\title{
LA MUJER RURAL: \\ EL CASO DE LAS VIRTUDES DE SANTA CRUZ DE TURRIALBA
}

\author{
RURAL WOMEN: \\ THE CASE OF LAS VIRTUDES IN SANTA CRUZ, TURRIALBA
}

\author{
Mayela Cubillo Mora* \\ Mario Alberto Sáenz Rojas**
}

\begin{abstract}
RESUMEN
En este artículo se analiza la participación de la mujer rural en el proceso productivo y reproductivo, la invisibilización de su trabajo en las unidades familiares agropecuarias y su rol reproductivo en una doble jornada de trabajo. Particularmente, en Las Virtudes de Santa Cruz de Turrialba se reconoce la importancia del grupo familiar en el desarrollo de esta comunidad, asimismo, en el estudio de sus integrantes se observa cómo se naturaliza ese rol de subordinación de la mujer rural, a pesar de trabajar en iguales condiciones que los otros miembros.
\end{abstract}

PALABRAS CLAVE: COSTA RICA * MUJERES * IDENTIDAD * ROL DE GÉNERO * PARTICIPACIÓN FEMENINA * SECTOR RURAL * FAMILIA

\section{ABSTRACT}

This article analyzes the participation of rural women in the productive and reproductive process, the invisibility of their work in agricultural households and their reproductive role in a double shift. Particularly, at Las Virtudes in Santa Cruz of Turrialba, the importance of family is recognized in the development of this community and the study of their members shows the naturalization of the subordinate role of rural women, despite working in the same conditions than men.

KEYWORDS: COSTA RICA * WOMENS * IDENTITY * GENDER ROLES * WOMENS PARTICIPATION

* RURAL SECTOR * FAMILY

* Centro de Investigación y Capacitación en Administración Pública (CICAP) de la Universidad de Costa Rica (UCR). mayela.cubillo@ucr.ac.cr

** Escuela de Psicología de la Universidad de Costa Rica (UCR), Sede de Guanacaste y Escuela de Psicología de la Universidad Católica de Costa Rica.

msaenzr@poder-judicial.go.cr 
Digo que yo trabajo, vivo, pienso, y que esto que yo hago es un buen rezo, que a Dios le gusta mucho y respondo por ello. Poema "Digo". Jorge Debravo

\section{INTRODUCCIÓN}

Diversas publicaciones señalan que Ester Boserup (1970) es la autora que realiza el primer estudio relevante sobre la participación femenina en el sector agropecuario de América Latina. Ella califica la agricultura latinoamericana como un "sistema agrario masculino", en el que la producción de alimentos es llevada a cabo fundamentalmente por los hombres, en contraposición a la agricultura africana, que la califica de "sistema agrario femenino".

Por su parte, en la agricultura andina, Carmen Deere y Magdalena León (2000), mencionan que en la década de los años 70 , existía un cierto grado de subordinación femenina como obligación, a pesar del aumento en la participación de la mujer en el mercado laboral. La contribución de su trabajo es la definición de una nueva categoría que denominaron "el sistema agrario de familia patriarcal". Este sistema consiste en que tanto los hombres como las mujeres aportan fuerza de trabajo, pero son los hombres quienes controlan el poder de decisión y el resultado de la producción.

En la década de 1980, se desarrolló una serie de estudios de caso que proporcionaron evidencia empírica sobre el rol significativo que desempeñaban las mujeres en la producción de las unidades agropecuarias familiares; por ejemplo, Carmen Deere realizó un análisis de las reformas agrarias en tres países de América Latina y determinó que es importante:

... incorporar a la mujer rural como socia en las empresas asociativas. Para asegurar la participación socio-económica de la mujer campesina, es imprescindible que las cooperativas no se constituyen solamente en base a los jefes de familia, sino que incorpora (sic) a todos los adultos (1983: 71)1.

Particularmente, en el caso de Costa Rica y en esta misma década, Carmen Romero, Mario Ramírez y Giannina Tanzi (1983) reconocían la relevancia del trabajo de la mujer en el ámbito de las actividades rurales, tanto productivas como reproductivas; asimismo, plantearon tres dimensiones, a saber: socio-espacial, socioeconómica e ideológica-cultural, para explicar la interrelación de procesos estructurales y coyunturales que influyen en la participación organizativa de la mujer rural.

Por otra parte, Mayra Achío y Patricia Mora (1988) señalaron que no existe un vínculo unidireccional y automático entre la incorporación de la mujer al proceso productivo de flores y la concienciación sobre su condición de subordinación: "una vez que la mujer ha salido del hogar a vender su fuerza de trabajo al capital, operan sobre ella todos los elementos de alienación que perturban al trabajador en general" (1988: 54).

En este mismo plano, Bernardo Bolaños y Hannia Rodríguez (1988) al estudiar el caso de la obrera florista, concluyeron que la subordinación de la mujer se agudiza y reproduce al incorporarse en el proceso productivo de corte capitalista, que abarca no solo el ámbito laboral en aspectos como la división sexual del trabajo y las diferencias salariales, sino también "la doble jornada de trabajo".

Posteriormente, en la década de 1990, Giselle Rodríguez, en una investigación para el Instituto Interamericano de Cooperación para la Agricultura-IIca (1996), desarrolló un estudio sobre "Mujeres Productoras de Alimentos" en 18 países de América Latina y el Caribe, en el cual se ratificó que las mujeres latinoamericanas desempeñan un papel muy importante en la producción agrícola.

En general, se entiende que el trabajo agrícola entre otras labores, considera el cultivo de la tierra, el cuidado del ganado y los trabajos del campo asociados con estas actividades;

\footnotetext{
$1 \quad$ Léase en su forma correcta: “...no se constituyan solamente con base en los jefes de familia, sino que incorporen a todos los adultos".
} 
además, tareas como la selección de semillas, almacenamiento, conservación y transformación de productos agrícolas, en las cuales las mujeres están frecuentemente involucradas.

Respecto al género, María A. Calvo (2003) menciona que es un término usado para referirse a las cualidades distintas del hombre $y$ la mujer que han sido creadas culturalmente; es decir, el género se presenta como rasgos de personalidad, actitudes, sentimientos, valores $y$ actividades que la sociedad describe formalmente como propias de hombres y mujeres. Esto se constituye en una idea construida históricamente, la cual se transforma con el tiempo a nivel macro y micro de las esferas sociales, mediante las instituciones e instancias de socialización, relaciones interpersonales y de las mismas autoimágenes individuales.

Estas características construidas socialmente, se generalizan de tal manera que las personas las asumen naturalmente, como parte de las diferencias genéticas entre sexos. Es así como cultural y socialmente se crea una jerarquización vertical de rasgos y actividades, teniendo mayor prestigio el hombre que la mujer.

El género es una construcción social en que los patrones socio culturales actúan para encasillar lo que se debe hacer y así lograr un supuesto orden dentro de la sociedad, que ya está concebida como un sistema donde todo se encuentra establecido y prediseñado pero si algún segmento rompe con lo ya predispuesto causa una crisis y desequilibrio, cuyas consecuencias se creen que son negativas (Calvo, 2003: 67).

Por otra parte, es necesario establecer la diferenciación conceptual entre sexo y género para abordar el tema de las mujeres. El sexo corresponde a un hecho biológico, producto de la diferencia sexual del ser humano, lo cual implica un proceso con distintos niveles, que no siempre coinciden entre sí y que son llamados por la biología y la medicina como sexo cromosómico, hormonal, anatómico y fisiológico.

Mientras tanto, el género viene a ser la percepción psicosocial que tiene la persona de su pertenencia sexual a partir de las atribuciones que la sociedad ha establecido para cada uno de los sexos individualmente constituidos (Gomáriz, 1994).

Según Laura Guzmán (1994), el género es además un instrumento que se propone explicar el impacto que tiene en cada persona el hecho de ser mujer $u$ hombre, en una sociedad determinada y en un momento histórico dado, pues lo femenino y lo masculino varían con el transcurrir del tiempo y de una cultura a otra, ya que son construcciones histórico-sociales.

Durante los primeros tres años de vida, las niñas y los niños aprenden a percibirse a sí mismas(os) como femeninas y masculinos, según la forma en que son estimulados cotidianamente por sus padres, familiares $u$ otras personas adultas significativas, así como por los medios de comunicación. Al respecto, señala acertadamente Mirta González:

Desde la primera infancia se motiva a los niños a expresar aspiraciones ocupacionales. Los preescolares tienen una comprensión bastante clara de la diferenciación del trabajo por sexo y tienden a decir sus preferencias por carreras tradicionales asociadas a su propio sexo. Estas preferencias se desarrollan tempranamente $y$ son evidentes mucho antes del ingreso a la escuela (1990: 48).

Lo anterior remite al concepto de socialización, expuesto en Martín-Baró (1999), mismo que alude a un proceso de desarrollo que es histórico y comprende tanto la identidad personal como la social. De esta manera, "en la socialización primaria, el individuo adquiere un mundo y desarrolla una identidad personal" (1999: 119). Esto implica, de conformidad con el mismo autor, una estructuración psicológica mediante la cual se adquieren un conjunto de esquemas cognitivos y se incorpora un marco valorativo que le sirve de referencia para interpretar la realidad.

Al ser una construcción social, el género no es algo inmutable sino que sufre modificaciones que van de la mano con los cambios culturales, sociales e históricos; asimismo, las personas pueden transformar sus comportamientos aprendidos; de manera que 
la categoría de género enfatiza las diferencias sociales entre lo masculino y lo femenino, que son contradictorias y cambiantes con el tiempo, aprendidas en un contexto mediante los procesos de socialización.

Por otro lado, Marcela Lagarde (2001a) expone que el patriarcado es una ideología que ha imperado durante siglos en la sociedad, esta ideología coloca en un plano inferior a la mujer, mientras que al hombre, lo posiciona como centro y referencia para todos los fenómenos sociales.

Para Lagarde, la ideología patriarcal es "una ideología fosilizada porque expresa y sintetiza separaciones simbólicas inmutables que no corresponden a la complejidad genérica de los sujetos. Su esencia consiste en elaborar las diferencias como excluyentes y antagónicas por naturaleza" (2001b: 6). Por ello, la realidad vivida por hombres y mujeres es captada desde la construcción de estereotipos, asimismo, ambos reciben penalizaciones sociales si no cumplen con el "deber ser" definido por la cultura hegemónica.

El patriarcado es la forma social que fundamenta la estereotipia de género. Así, las relaciones de poder del patriarcado han difundido la diferenciación de tareas asignadas a hombres $y$ mujeres; para ellos, lo concerniente a la esfera pública y para ellas, lo relacionado con la esfera de lo privado, el hogar.

En otras palabras, a los hombres se les destinan las actividades y los trabajos públicos de transformación, incremento o destrucción de vidas humanas, del medio, los territorios y los bienes. Asimismo, la creación y mantenimiento de las concepciones de mundo; es decir, las creencias, los valores, las sabidurías y las obras culturales (Lagarde, 1997).

Lo anterior es reafirmado por Enrique Gomáriz (1994), quien explica cómo la sociedad ha construido una serie de prácticas, representaciones, normas $y$ valores específicos para cada sexo, que condicionan y guían su forma de ser. De este modo, la identidad de cada cual se elabora en espacios sociales diferentes, tanto a nivel privado como público.

En este sentido, Martín-Baró (1999) plantea que al síndrome del machismo corresponde a lo que se denomina síndrome del "hembrismo", caracterizado por la subordinación de carácter instrumental frente al macho, la exigencia de virginidad $y$ enclaustramiento, la dedicación a la sensibilidad y afectividad, por último, el conservadurismo y la religiosidad. De acuerdo con este autor, este síndrome describe acertadamente en mayor o menor grado, a la mayoría de mujeres en el medio latinoamericano.

A pesar de las políticas de equidad y al avance en esta materia, sigue existiendo mayoría de hombres que ejercen los cargos de poder en las organizaciones sociales, dándose una posición privilegiada de lo masculino y una inferioridad de las mujeres y lo femenino, sobre todo en espacios de dominación, como son la familia y la comunidad.

Según Lagarde (2001b), el enfoque de género nace como una alternativa orientada a realizar análisis de fenómenos sociales, políticos, económicos y culturales considerando como primer punto, las construcciones sociales de género. Asimismo, permite observar la realidad tomando como base las variables sexo y género, así como, las formas en que estas se manifiestan en un contexto geográfico, cultural, étnico e histórico determinado.

En este contexto, interesa observar si hay reconocimiento por parte de la sociedad $y$ de las mismas mujeres, cuál es la contribución que las mujeres realizan a la producción agropecuaria, a la reproducción de la familia, a su mantenimiento y fortalecimiento, así como su participación en la solución de los problemas a los que se enfrentan en su entorno y a la construcción misma de un pueblo.

\section{METODOLOGÍA}

Para ello, se planteó la realización de una investigación de carácter etnográfico como técnica de investigación. El método etnográfico conlleva la observación participante o las entrevistas a las personas o grupos durante un cierto espacio de tiempo. Esta relación "cara a cara" y las respuestas a las entrevistas permiten conocer desde su posicionamiento, lo que sienten o piensan acerca de su comunidad, las relaciones con sus vecinos, familia, los problemas que les aquejan y su visión de mundo. Esta técnica 
permite aprehender el modo de vida de una unidad social concreta ${ }^{2}$.

Miguel Martínez define el método etnográfico como “... la producción de estudios analítico descriptivos de las costumbres, creencias, prácticas sociales $y$ religiosas, conocimientos y comportamiento de una cultura particular, generalmente de pueblos o tribus primitivos" (1999: 109).

De una manera menos restringida y siguiendo un sentido más amplio, Goetz y LeCompte plantean varios aspectos conceptuales importantes de destacar respecto a lo que es la etnografía:

$\diamond \quad$ “... una descripción o reconstrucción analítica de escenarios y grupos culturales intactos",

$\diamond \quad$ un método que revive "... para el lector las creencias compartidas, prácticas, artefactos, conocimiento popular y comportamientos de un grupo de personas",

$\diamond \quad$ una disciplina que “... comienza examinando grupos $y$ procesos incluso muy comunes, como si fueran excepcionales o únicos",

$\diamond \quad$ una técnica que “... permite apreciar los aspectos, tanto generales como de detalle, necesarios para dar credibilidad a su descripción" (1988: 28).

El presente estudio se realizó en la comunidad de Las Virtudes de Santa Cruz de Turrialba, por la accesibilidad de la comunidad a participar como objeto de investigación.

Se realizaron tres visitas, en las que se convivió con la familia A.-F., por medio de la cual se tuvo acceso al resto de los miembros de la comunidad.

2 "Etimológicamente el término etnografía proviene del griego "ethnos" (tribu, pueblo) y de "grapho" (yo escribo) y se utiliza para referirse a la descripción del modo de vida de un grupo de individuos". (Woods, 1987 citado en Barbolla, Benavente, López, Martín, Perlado y Serrano, 2010. Consultar: <http://www.uam.es/personal_pdi/stmaria/jmurillo/InvestigacioneE/Presentaciones/Curso_10/I_ Etnografica_Trabajo.pdf>
Asimismo, se efectuaron varias entrevistas en profundidad con líderes de la comunidad $y$ con las mujeres de la familia A.-F.

\section{LA FAMILIA A.-F. COMO EJEMPLO DE BASTIÓN DE UNA COMUNIDAD ${ }^{3}$}

Doña Flor inició su historia narrando que es oriunda de Bonilla de Santa Cruz y que se casó a los 17 años de edad con Santiago A., un 16 de diciembre, para que le ayudara con su "enfermedad" (refiriéndose al período menstrual), ya que su familia era muy pobre y no tenía posibilidades de pagarle atención médica para curarla.

Doña Flor comentó que no tuvo noviazgo, debido al conservadurismo de sus padres $y$ que la única diversión que tenía "era ir a misa". Ahí conoció a Santiago. Sus padres no la dejaban pintarse ni ponerse pantalones, pero ella a sus hijas sí se los permitió.

Sus padres eran campesinos y mientras estuvo soltera, siguió los pasos de su madre, de quien aprendió todos los oficios domésticos. Pero fue a la par de su esposo que se inició en las labores de sembrar la tierra, cuidar el ganado, "desmatorrar" pastos, ordeñar, hacer y comercializar el queso, entre otras actividades. Todo ello, sin dejar de atender sus labores de madre y esposa, las cuales solo dejó de lado durante sus seis partos.

Doña Flor indicó que todos sus hijos nacieron en Bonilla, fueron seis y los dos mayores son varones. Entre los primeros tres, había dos años de distancia aproximadamente, pero con las restantes, la diferencia en años fue mayor (alrededor de cinco años).

Asimismo, mencionó que la decisión de irse de Bonilla hacia Las Virtudes y comprar una finca, fue de Santiago. Ella nunca participó de la decisión, se limitó a seguirlo junto con sus cinco hijos, ya que el mayor se quedó en Bonilla hasta que vendieron la finca que tenían en ese lugar: "él me trajo a ver la finca que había comprado y me gustó".

En Bonilla, tenían también un terreno donde cultivaban café y caña, pero él quería

3 Se anotan solo las iniciales, con el objeto de mantener el anonimato de la familia. 
una finca más grande y por ello salieron de ahí: "cuando mi esposo salió de Bonilla, empezaron a salir todos sus hermanos".

El traslado de Bonilla hasta Las Virtudes significó un cambio en la situación de la familia, ya que de una casa amplia con luz y agua se trasladaron a vivir a un galerón con cuatro paredes nada más. Inicialmente, las necesidades fisiológicas las hacían en el monte.

Virgita, la hija mayor, recordó que ella trabaja desde los 11 años de edad, salía de la escuela descalza para ayudar a la familia. Para el momento del traslado a Las Virtudes, ella contaba con 16 años de edad y expresó que "fue un cambio muy demasiado grande", pasaron "de tener electricidad y casa cómoda", a una galera, fue un cambio "como del cielo a la tierra".

Virgita agregó que desde niña (4-5 años) sus padres se iban a recolectar café a las 5 am, mientras que los hijos mayores se iban a ordeñar. En su familia "lo mismo trabajan los hombres que las mujeres $y$ la plata la maneja el núcleo familiar". Al profundizar en esta afirmación indica que "lo gastaban entre todos", pero el dinero lo manejaba el padre.

Doña Flor mencionó que ella se dedicaba a sembrar papa, "desmatorrar" potreros, picar leña, mientras la hija mayor a veces le ayudaba en estas tareas o se quedaba en la casa para atender a las hermanas menores.

El rol de la hija mayor es siempre de más responsabilidad, trabajo duro y rigor de los padres, en comparación con el resto de la prole. Virgita debió asumir desde niña los trabajos domésticos, el cuido y la atención de sus hermanos menores, mientras la madre se dedicaba junto con el padre, a los trabajos del campo.

Actualmente, todas las mujeres de la familia trabajan en las labores propias de la producción lechera, las cuales inician a tempranas horas del día, aún cuando la oscuridad domina el paisaje. Realizan el ordeño de las vacas, alimentan al ganado, cortan y trasladan el pasto; paralelo a ello cumplen con las tareas domésticas: "Después del ordeño, nos trasladamos de la lechería a preparar el desayuno para la familia $y$ alistar los hijos para enviarlos a la escuela. Y luego de algunas labores domésticas, llega la hora de preparar el queso".
El trabajo de ordeño y cuido del ganado es compartido con los varones en igualdad de condiciones y se realiza en dos turnos: con el alba y luego continúa en las primeras horas de la tarde, cuando se realiza el segundo ordeño del día. Este ciclo productivo nunca se interrumpe: "esta jornada laboral es de nunca acabar. No hay domingos, ni días feriados, ni vacaciones, ni aguaceros, ni tormentas".

Aunado a este horario de trabajo, las mujeres se encargan de las actividades relacionadas con el hogar y la crianza de los hijos y las hijas.

Gabriela, una de las hijas menores del matrimonio indica que ella trabaja de 3 a 4 de la mañana en las labores de ordeño, en la elaboración del queso de 10-11 de la mañana y de 2 a 3 de la tarde, en el segundo ordeño del día; el resto del día lo utiliza en la atención de los hijos $y$ las labores domésticas. En tono de broma (pero no hay chiste sin verdad) menciona que "mi esposo ni siquiera sabe que sus hijos van a la escuela".

Virgita, que vive actualmente en el centro de Turrialba $y$ tiene una soda $y$ una tiendita de quesos, indicó que a sus hijos les interesaban más las labores de la finca que la escuela, y le decían "mami no me mande más a la escuela". Es por ello que estos siguen trabajando el campo en la finca familiar de Las Virtudes.

Por su parte, Gabriela opina al igual que sus hermanas y su madre que económicamente, no les hubiera ido bien, si el trabajo en la finca no incluyera a toda la familia.

Las tres hijas presentes opinan y concuerdan en que el papel de ellas y el de su madre no ha variado con el tiempo y la única diferencia estriba en que ahora se tienen mayores comodidades en la casa. Se refieren al agua, electricidad, artefactos electrodomésticos y televisión, entre otras.

Respecto al trabajo doméstico que socialmente no es remunerado, ni reconocido, ni contabilizado en la economía familiar, en este caso tampoco lo es el trabajo de la mujer en las lecherías o en la confección del queso. De esta manera, el trabajo de la mujer es doblemente devaluado e invisibilizado $y$ a pesar de que, como dijo don Narciso, "a veces trabajan mejor 
que un hombre" (líder comunal, entrevista, 24/03/2012).

Esto evidencia el sometimiento de la mujer a una doble jornada de trabajo, bajo un régimen, en el cual no es reconocido como tal $y$ por lo tanto, no reciben ningún ingreso por sus labores.

La familia A.-F. representa una economía familiar donde todos sus miembros trabajan como unidad productiva, pero es el hombre el que dispone cómo se distribuye el ingreso familiar, al igual que cuánto y cómo se utiliza, tanto para el consumo como para la inversión.

Refiriéndose a los ingresos producto de la venta de la leche, el queso y la natilla, Virgita comentó: "la plata la maneja el núcleo familiar" (entrevista, 24/03/2012). Lo cierto es que esta última frase alude a la manutención económica del hogar pero nunca a que la mujer disponga de su propio dinero o maneje lo que se ha recaudado en conjunto, puesto que eso queda en manos del hombre y resulta algo incuestionable para las mujeres.

Esta realidad es difícil de comprender si no se alude al proceso de socialización femenina, incluyendo los valores, los estereotipos y los mitos que se le inculcan a las mujeres sobre lo que es "ser mujer", particularmente, la subordinación al hombre, el enclaustramiento en el hogar, la sensibilidad y el conservadurismo.

Entre los valores que aprenden las mujeres de la familia se encuentra la imagen del padre-esposo como una figura de autoridad atemorizante, cuyas decisiones no son rebatidas ni cuestionadas. De esta manera, si las mujeres son subordinadas, el padre es sometedor, evidenciando un protovínculo social de dominación. Se puede afirmar que el padre sometió a sus hijas a una dinámica de control social de la sexualidad por medio de las múltiples restricciones impuestas al noviazgo de estas, imposición que aún hoy es resentida por Virgita.

Esta incuestionabilidad de las decisiones del hombre y el ejercicio de su autoridad es a menudo disimulada, bajando el volumen de la voz cuando el padre y esposo se acerca o como señaló doña Betty, una de las vecinas de Las Virtudes $y$ dirigente comunal: "yo integraba al marido a las actividades comunales, ya que en mi pensamiento estaba si mi esposo se iba a molestar porque yo dedicaba tanto tiempo a los proyectos en la comunidad" (entrevista, 24/03/2012).

\section{LAS MUJERES Y EL DESARROLLO DE LA COMUNIDAD DE LAS VIRTUDES}

Los vecinos narran que al inicio la comunidad se llamaba "Raiceros" o "Peor es Nada", debido a que el lugar era montaña y potreros perdidos. Con este nombre aparece en el Registro de la Propiedad.

La comunidad de Las Virtudes, hoy dividida en dos partes, de las cuales una parte perteneció a una finca que se llamaba Las Virtudes, cuyo propietario fue don Florentino Castro. Este señor fue una especie de patriarca que tuvo muchos hijos y ostentó posiciones de poder económico y grandes vinculaciones políticas. La otra parte de la comunidad que hoy se llama Calle Vargas, perteneció a don Lucas Vargas (bisabuelo de doña Betty y de la esposa de don Narciso). La parte alta de esta finca fue parcelada por el Instituto de Desarrollo Agrario (IDA) y cedida a varios beneficiarios.

La primera construcción institucional que se hizo en el pueblo fue la escuela, por iniciativa de sus habitantes (1970), utilizando una casa como inmueble. Luego se construyó el templo católico. Al respecto, comentó doña Betty "que primero se dio la escuela y luego la ermita porque la misa se daba en la escuela y era muy pequeña e incómoda" (entrevista, 24/03/2012).

Para la construcción de la ermita, primero consiguieron un lote que fue donado por José Narciso Casasola y se formó un Comité de Actividades conformado por un grupo de jóvenes (entre ellas tres mujeres: Dora Emilia Ulloa, Yenory Solano y Betty Camacho). Inicialmente, este grupo era grande $y$ paulatinamente se fue reduciendo. Las personas integrantes de este comité recogían dinero y organizaban diversas actividades para recaudar fondos, tales como un reinado, rifas, ferias $y$ visitas al Ministerio de Obras Públicas y Transportes para conseguir donaciones de materiales. Con estos aportes se construyó el templo y se designó como patrono a San José, en honor al donador del terreno. 
Posteriormente, se construyó el salón comunal en el mismo lote de la ermita, con una ampliación de la donación. Don Narciso era la persona con mayores recursos económicos del pueblo, asimismo, se caracterizaba por su espíritu caritativo, apoyando las obras que impulsan el desarrollo de la comunidad.

Seguidamente, se dio la construcción de la plaza, el asfaltado de las calles y la construcción del puente, que se realizó mediante el trabajo de la Asociación de Desarrollo Comunal. Todos expresan que el asfaltado de la calle se debió al aporte de Ovidio Pacheco, oriundo del lugar, cuando fue diputado por el Partido Unidad Social Cristiana, a quien le agradecen, pero que también le resienten que no se concluyera y se dejara la finalización de la obra al partido opositor, Liberación Nacional, obra que a la fecha no ha concluido.

Esta construcción de la calle es vital para el desarrollo del pueblo, ya que ellos mismos expresan que "al inicio no había caminos, para ir a Turrialba a vender el queso se tardaban 8 horas a caballo ida y regreso". Sin caminos no era posible comercializar lo que producían, por ello la calle y el puente construidos entre 19861988, significan un antes y un después para la comunidad de Las Virtudes.

Es importante mencionar que existe un reconocimiento de las personas del pueblo a don Santiago A. y a su hijo mayor, Glenn. Con la llegada de la familia A.-F., todas las obras de infraestructura de la comunidad se remodelaron. Ellos "invirtieron tiempo y dinero para mejorar la construcción de la iglesia, la escuela, el salón comunal y las calles", comentó don Narciso (24/03/2012).

Las mujeres jugaron un papel protagónico en la movilización para la construcción de la ermita y también colaboraron en la escuela, en actividades como limpieza, cocina y el patronato escolar.

Además, las mujeres tienen una significativa participación en la organización comunitaria, no ostentando cargos en los comités creados para cumplir distintos fines (construcción de la ermita, del salón comunal, patronato escolar, entre otros), sino también, desempeñando un papel activo y protagónico en la recolección de fondos mediante la modalidad "puerta por puerta" o visitando la Municipalidad y diferentes instituciones públicas, con el fin de solicitar apoyos financieros o materiales, al igual que trasladando una actividad doméstica específica, como es la cocina, a los festejos comunales.

En la construcción del pueblo, las mujeres trabajaron junto a los hombres $y$ en la medida en que la comunidad de Las Virtudes creció en número de habitantes, se realizó un mejoramiento de la calidad de vida mediante el acceso a servicios públicos (calles, escuela y salón comunal); es decir, en todo este proceso, las mujeres hicieron historia pero los hombres son quienes la cuentan desde su perspectiva $y$ son sus nombres los que perduran, lo cual es muestra de su posición de dominio.

En Las Virtudes, la mayor parte de las mujeres se dedican solamente al trabajo del hogar, mientras que doña Flor y sus hijas trabajan "hombro a hombro" junto con los hombres. Ellas mencionan que en la comunidad se refieren a ellas como "las vaqueras". Al respecto, Virgita expresó que "las costumbres de trabajar en el campo ya las traían desde que vivían en Bonilla $y$ al llegar a Las Virtudes no las dejaron" (entrevista, 24/03/2012).

Respecto al futuro de su comunidad, las personas mayores no se sienten optimistas. Hablan de la apatía de la juventud actual en torno a la participación social, "la gente joven no quiere participar", incluso, don Narciso comentó que vendió sus tierras y animales porque a sus nietos no les interesa dar continuidad a su legado.

\section{REFLEXIONES FINALES: APUNTES PARA UN NUEVO COMIENZO}

En la comunidad de Las Virtudes de Santa Cruz de Turrialba, la mujer ha desempeñado $y$ posee actualmente, un papel fundamental en el sistema productivo y en la reproducción de la fuerza de trabajo.

En cuanto a la división del trabajo que se establece entre hombres $y$ mujeres rurales en la agricultura familiar de Las Virtudes, se pueden identificar varias generalidades: 
1) Existe una combinación o mezcla entre la unidad familiar de producción (finca) y la unidad doméstica (casa), donde todas $y$ todos los miembros de la familia tienen participación en su funcionamiento, aunque esta puede darse en grado diverso.

2) No existe división en la gestión de la unidad de producción y la gestión de la unidad familiar; es decir, el proceso de toma de decisiones no diferencia la forma de combinar los recursos productivos, la administración del establecimiento y las decisiones familiares.

3) Es evidente el carácter patriarcal de la familia A.-F. y de la comunidad en general, en la que se atribuye al hombre el papel de proveedor de la familia, invisivilizando el trabajo femenino y reforzando la subordinación de la mujer.

4) Si bien, las mujeres de la familia participan en la esfera productiva de las unidades familiares agropecuarias, no son reconocidas ni valoradas socialmente, aspecto que es aceptado y naturalizado por la ideología que a ellas mismas las interpela.

5) La familia A.-F. se instala en Las Virtudes $y$ con el trabajo de todos ellos comienza su historia de éxito. Su ascenso se da cuando se construyeron las vías de acceso para sacar los productos, adquieren sus propios medios de transporte e inician el proceso de comercializar el queso en las Ferias del Agricultor.

6) Gran parte de la historia reciente de la comunidad de Las Virtudes y de sus principales logros en infraestructura y educativos se debe a la llegada de la familia A.-F.

7) Las mujeres de Las Virtudes se autocalifican como trabajadoras del hogar, lo cual coadyuva a que estas no sean incluidas dentro de la población económicamente activa (PEA), reafirmando su rol de "doble jornada de trabajo", lo cual obstaculiza sus posibilidades de toma de conciencia en torno a su condición de subordinación.

8) El trabajo de las mujeres de Las Virtudes en la producción lechera y sus derivados no es remunerado. Esta falta de remuneración, la fragmentación del trabajo doméstico con el de unidad de producción y la percepción de ellas mismas sobre el escaso valor monetario de su trabajo, son todos elementos que contribuyen a la sub-valoración del trabajo de la mujer rural y a su invisibilización.

9) Por último, la familia A.-F. es percibida en la comunidad como la representación del ideal social-patriarcal de un modelo familiar (nuclear-conyugal).

\section{BIBLIOGRAFIA}

Achío, Mayra y Mora, Patricia. "La obrera florista y la subordinación de la mujer". Revista de Ciencias Sociales 39. San José, Costa Rica. Editorial de la Universidad de Costa Rica, 1988: 47-56.

Barbolla Diz, Cristina; Benavente Martínez, Nuria; López Barrera, Tamara; Martín de Almagro Gómez, Cristina; Perlado Sotodosos, Lucía y Serrano de Luca, Carme. Investigación etnográfica. 2010. En: http://www.uam.es/personal_pdi/ stmaria/jmurillo/Investigacionee/ Presentaciones/Curso_ $10 / I_{-}$ Etnografica_Trabajo.pdf [consultado el 18 de noviembre de 2014].

Bolaños, Bernardo y Rodríguez, Hannia. "La incorporación de la mujer en el proceso productivo de flores en Costa Rica”. Revista de Ciencias Sociales 39. San José, Costa Rica. Editorial de la Universidad de Costa Rica, 1988: 57-68.

Boserup, Ester. Woman's rol in economics development. New York, eEuu: St Martin's, 1970.

Calvo, María Antonietta. "Mujeres con discapacidad: sus percepciones y su vida cotidiana. El caso de 14 mujeres". [Tesis de Magister en Estudios Interdisciplinarios sobre Discapacidad]. Universidad de Costa Rica, 2003.

Deere, Carmen Diana. "La mujer rural y las reformas agrarias en Perú, Chile y Cuba”. Revista de Ciencias Sociales 25. San José, Costa Rica. Editorial de la Universidad de Costa Rica, 1983: 59-74. 
Deere, Carmen Diana y León, Magdalena. Género, propiedad y empoderamiento: tierra, Estado y mercado en América Latina. Bogotá, Colombia: Tercer Mundo, 2000.

Goetz, J. P. y LeCompte, M. D. Etnografía y diseño cualitativo en investigación cualitativa. Madrid, España: Morata, 1988.

Gomáriz, Enrique. La planificación con perspectiva de género. San José: Centro Nacional para el Desarrollo de la Mujer y la Familia, 1994.

González Suárez, Mirta. El sexismo en la educación. San José, Costa Rica: Universidad de Costa Rica, 1990.

Guzmán Stein, Laura. "Relaciones de género y estructuras familiares". Revista Costarricense de Trabajo Social 4. San José, Costa Rica: Colegio de Trabajadores Sociales de Costa Rica, diciembre 1994.

Lagarde, Marcela. "Género y feminismo. Desarrollo Humano y democracia". Cuadernos Inacabados. 2nda. edición. España: Horas y Horas, 1997.

Lagarde, Marcela. Claves feministas para la negociación en el amor. Managua, Nicaragua: Puntos de Encuentro, 2001a.

Lagarde, Marcela. "Poder, relaciones genéricas e interculturales". Actas de conferencias internacionales. Primer Encuentro Mesoamericano de Estudios De Género: Colección Estudios de Género 5. Antigua Guatemala, Guatemala. Facultad
Latinoamericana de Ciencias Sociales, 2001b: 13-50.

Martín-Baró, Ignacio. Acción e ideología. Psicología social desde Centroamérica. 9na. edición. San Salvador, El Salvador: Universidad Centroamericana, 1999.

Martínez, Miguel. Comportamiento humano; nuevos métodos de investigación. 2nda. edición. México DF: Trillas, 1999.

Rodríguez, Giselle. La mujeres productoras de alimentos en Costa Rica: tecnología y comercialización. San José: IIcA, 1996.

Romero, Carmen María; Ramírez, Mario y Tanzi, Giannina. "La investigación de los problemas de la mujer rural". Revista de Ciencias Sociales 25. San José, Costa Rica. Editorial de la Universidad de Costa Rica, 1983: 47-58.

\section{ENTREVISTAS}

Betty Camacho. Líder comunal de Las Virtudes. 24 de marzo de 2012.

Flor F. Madre de familia A.-F. 24 de marzo de 2012.

Narciso Casasola. Líder comunal. 24 de marzo de 2012.

Santiago A. Padre de familia A.-F. 24 de marzo de 2012.

Virgita, Gabriela y Sileny. Hijas de familia A.-F. 24 de marzo de 2012.

Fecha de ingreso: 02/07/2013 Fecha de aprobación: 23/10/2013 